Article

\title{
Mucoadhesive Electrospun Nanofiber-Based Hybrid System with Controlled and Unidirectional Release of Desmopressin
}

\author{
Mai Bay Stie ${ }^{1,2} \mathbb{D}$, Johan Ring Gätke ${ }^{1,2}$, Ioannis S. Chronakis ${ }^{3} \mathbb{D}$, Jette Jacobsen ${ }^{1}$ and Hanne Mørck Nielsen ${ }^{1,2} *^{(\mathbb{D}}$ \\ 1 Department of Pharmacy, University of Copenhagen, Universitetsparken 2, 2100 Copenhagen, Denmark; \\ mai.bay.stie@sund.ku.dk (M.B.S.); kjn376@alumni.ku.dk (J.R.G.); jette.jacobsen@sund.ku.dk (J.J.) \\ 2 Center for Biopharmaceuticals and Biobarriers in Drug Delivery, Department of Pharmacy, \\ University of Copenhagen, Universitetsparken 2, 2100 Copenhagen, Denmark \\ 3 DTU-Food, Technical University of Denmark, Kemitorvet, B202, 2800 Kongens Lyngby, Denmark; \\ ioach@food.dtu.dk \\ * Correspondence: hanne.morck@sund.ku.dk
}

check for

updates

Citation: Stie, M.B.; Gätke, J.R.;

Chronakis, I.S.; Jacobsen, J.; Nielsen, H.M. Mucoadhesive Electrospun Nanofiber-Based Hybrid System with Controlled and Unidirectional

Release of Desmopressin. Int. J. Mol. Sci. 2022, 23, 1458. https://doi.org/ $10.3390 /$ ijms 23031458

Academic Editor: Raghvendra

Singh Yadav

Received: 20 December 2021

Accepted: 23 January 2022

Published: 27 January 2022

Publisher's Note: MDPI stays neutral with regard to jurisdictional claims in published maps and institutional affiliations.

Copyright: (C) 2022 by the authors. Licensee MDPI, Basel, Switzerland. This article is an open access article distributed under the terms and conditions of the Creative Commons Attribution (CC BY) license (https:// creativecommons.org/licenses/by/ $4.0 /)$

\begin{abstract}
The sublingual mucosa is an attractive route for drug delivery, although challenged by a continuous flow of saliva that leads to a loss of drug by swallowing. It is of great benefit that drugs absorbed across the sublingual mucosa avoid exposure to the harsh environment of the gastro-intestinal lumen; this is especially beneficial for drugs of low physicochemical stability such as therapeutic peptides. In this study, a two-layered hybrid drug delivery system was developed for the sublingual delivery of the therapeutic peptide desmopressin. It consisted of peptide-loaded mucoadhesive electrospun chitosan/polyethylene oxide-based nanofibers (mean diameter of $183 \pm 20 \mathrm{~nm}$ ) and a saliva-repelling backing film to promote unidirectional release towards the mucosa. Desmopressin was released from the nanofiber-based hybrid system (approximately $80 \%$ of the loaded peptide was released within $45 \mathrm{~min}$ ) in a unidirectional manner in vitro. Importantly, the nanofiber-film hybrid system protected the peptide from wash-out, as demonstrated in an ex vivo flow retention model with porcine sublingual mucosal tissue. Approximately $90 \%$ of the loaded desmopressin was retained at the surface of the ex vivo porcine sublingual mucosa after $15 \mathrm{~min}$ of exposure to flow rates representing salivary flow.
\end{abstract}

Keywords: sublingual delivery; biopharmaceuticals; peptide drug delivery; electrospinning; mucoadhesion; ex vivo flow retention model

\section{Introduction}

Biopharmaceuticals-e.g., peptides and proteins-are the fastest growing group of drugs [1]. Peptides and proteins are highly potent and specific, but suffer from a low physicochemical stability; poor absorption across biological membranes; and, as a consequence, low bioavailability [2]. The primary route of administration of peptides and proteins is therefore via injections, which is inconvenient and often associated with poor patient compliance; new approaches for non-injectable formulations of peptides and proteins are therefore needed. The sublingual route is an attractive, non-invasive alternative site of administration as the mucosa is non-keratinized, highly vascularized, and consists of only 8-12 cell layers and thus has a reasonably low thickness $(100-200 \mu \mathrm{m})$ compared to the much thicker buccal mucosa [3]. Furthermore, drugs delivered via the oral cavity avoid the harsh environment of the gastro-intestinal tract and bypass the hepatic firstpass metabolism. These factors are beneficial for drugs, which are prone to enzymatic degradation and sensitive to the low $\mathrm{pH}$ of the stomach [4]. Formulations for sublingual administration can easily be handled by the patient as the ventral side of the tongue is readily accessible and the quick removal of the formulation is possible if needed. Further, such formulations can be administered without water, which is beneficial for patient groups with swallowing difficulties—e.g., the elderly or small children [5]. 
Sublingual administration of peptides and proteins is challenged by a continuous flow of saliva (total volume of saliva of 0.5-2 L/day) that may lead to the swallowing of the drug or drug delivery system and the subsequent degradation of the drug in the gastro-intestinal tract [6]. Even if not swallowed, the dilution of drug in the saliva impairs absorption by decreasing the amount of drug available at the site of absorption and thus also the drug concentration gradient across the mucosal epithelium, the most significant biological barrier to the absorption of the drug. Through a rational design, mucoadhesive drug delivery systems can protect the drug by encapsulation, retain the drug at the site of application for a prolonged period by controlled drug release, and thus not only increase, but also maintain the drug concentration gradient across the tissue, leading to the improved absorption of drug and therefore higher systemic bioavailability.

Electrospun nanofibers are especially suitable for topical administration on, e.g., the sublingual mucosa because of their high surface area to volume ratio and tunable mechanical properties, which can make them flexible enough to bend to the curved mucosal surfaces in the mouth. We have previously demonstrated a robust protocol for the electrospinning of biocompatible chitosan/polyethylene oxide (PEO) nanofibers [7] and demonstrated their suitability for sublingual administration because of their mucoadhesive properties [8]. The use of electrospun nanofibers has been explored for the oromucosal delivery of peptides and proteins, including insulin [9-11], FITC-labelled albumin [12], and lysozyme [13]. Reports aiming to achieve peptide delivery by the sublingual route are rare [9].

The therapeutic peptide desmopressin is used for the treatment of, amongst others, nocturnal enuresis, diabetes insipidus and hemophilia A. This peptide is currently administered either as a solution by intravenous or subcutaneous injection, as a nasal spray, or as a freeze-dried sublingual tablet. The bioavailability of desmopressin after sublingual administration of a freeze-dried fast-dissolving tablet is, however, reported to be only $\sim 0.25 \%$ [14], leaving room for improvement. Desmopressin is a relatively small peptide; it is modified from the structure of vasopressin (an endogenous hormone with a half-life in plasma of 10-35 $\mathrm{min}$ ) to display a higher stability (half-life in plasma 160 $\mathrm{min}$ ) and 10 times higher antidiuretic potency and 1500 times lower vasoconstricting potency compared to its natural analogue [15].

In this work, a combination of mucoadhesive electrospun chitosan/PEO nanofibers that facilitate the controlled release of desmopressin and a local high concentration at the site of application, and a water-repelling backing film that ensures unidirectional release and prevents the wash-out of peptide by saliva, were assessed for the sublingual delivery of the therapeutic peptide desmopressin. The protection of desmopressin from wash-out by saliva subsequent to the sublingual administration of the nanofiber-film hybrid system was also evaluated ex vivo.

\section{Results and Discussion}

\subsection{Clinically Relevant Doses of Desmopressin Loaded in Nanofiber-Film Hybrid System}

A two-layered hybrid drug delivery system, which consisted of a mucoadhesive electrospun chitosan-based nanofiber mat and a saliva-repelling backing film (Figure 1a), was developed. It is important to note that the peptide delivery system is considered biocompatible, as all selected excipients are biocompatible, and the electrospun nanofibers were prepared using a minimum amount of acetic acid $(0.7 \%(w / w))$. The therapeutic peptide desmopressin was encapsulated within the nanofibers by co-electrospinning. Then, a saliva-repelling backing layer was sprayed onto the fiber mat as a thin film based on ethyl-cellulose. The food coloring iron oxide, a red pigment insoluble in water, was also included in the film to provide a visual discrepancy in the orientation of the nanofiber-film hybrid system (Figure 1b). As visualized by scanning electron microscopy (SEM), close connection between the fibers and the thin coherent film was achieved (Figure 1c). The peptide-loaded nanofibers were smooth, uniform, and without artifacts such as beading (Figure 1d), with an average diameter of $183 \pm 20 \mathrm{~nm}$ (mean \pm standard deviation (SD)) and with a narrow size distribution. The average diameter of the nanofibers is similar 
$(168 \pm 38 \mathrm{~nm})$ to that of electrospun chitosan/PEO nanofibers prepared under the same conditions without the peptide [8].

The loading of desmopressin in the nanofibers was $8 \%(w / w)$. The weight of a $10 \mathrm{~mm}$ nanofiber disc (Figure $1 \mathrm{~b}$ ) was in the range of $1.5-3 \mathrm{mg}$; hence, the fiber discs provide a theoretical loading of desmopressin of 120-240 $\mu \mathrm{g}$ per disc, which is equivalent to the dose of desmopressin in the freeze-dried, sublingual tablets already on the market. Marketed freeze-dried tablets with desmopressin for sublingual administration (Minirin ${ }^{\circledR}$ by Ferring Pharmaceuticals) contain 60,120, or $240 \mu \mathrm{g}$ of desmopressin per dose. Thus, clinically relevant doses of desmopressin per nanofiber patch were achieved.

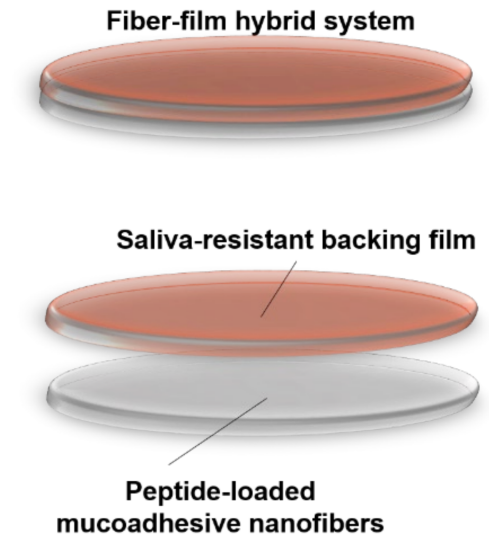

(a)

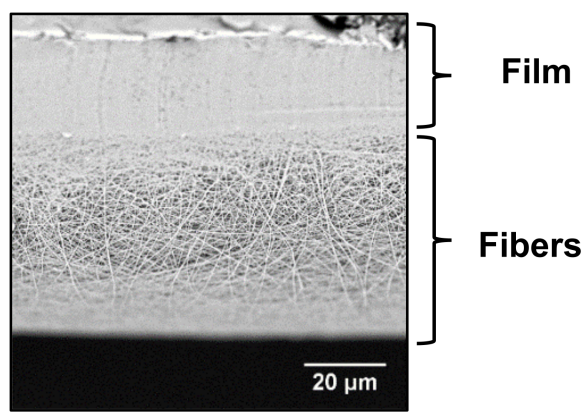

(c)

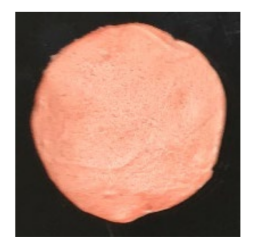

Film

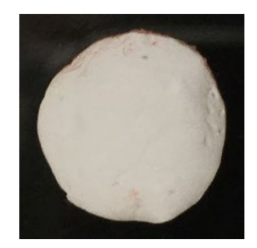

(b)
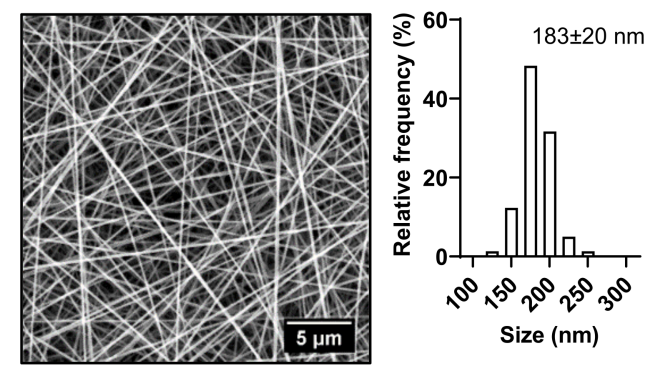

(d)

Figure 1. Properties of the desmopressin-loaded nanofiber-film hybrid system. (a) Schematic representation of the nanofiber-film hybrid system. (b) Image of a $10 \mathrm{~mm}$ disc of the two-sided nanofiber-film hybrid system. (c) Cross-section of peptide-loaded nanofiber-film hybrid system visualized by SEM. (d) Desmopressin-loaded chitosan/PEO nanofibers visualized by SEM. The size distribution of the nanofibers is given. The diameter is presented as mean $\pm \mathrm{SD} . N=3, n=100$, where $N$ is the number of individual batches of nanofibers produced, and $n$ is the number of nanofibers measured per batch.

\subsection{Nanofiber-Film Hybrid System Ensures Controlled and Unidirectional Release of Desmopressin}

A thin water-repelling backing film was applied to the mucoadhesive nanofiber layer to facilitate the unidirectional release of desmopressin, and, furthermore, to protect the drug delivery system and released therapeutic peptide from wash-out by saliva. The unidirectional and controlled release of desmopressin from the nanofibers and the barrier function of the water-repelling backing film were evaluated by determining the release of desmopressin into both sides of a diffusion cell (Ussing chamber) separated by the nanofiber-film hybrid system (Figure 2a). In the absence of the backing-membrane used as a control, approximately $50 \%$ of the loaded peptide was released from the nanofibers in the left and right chambers-i.e., it did not show a unidirectional release. We have previously 
demonstrated that electrospun chitosan/PEO nanofibers display extraordinary swelling properties and can swell $>1000 \%(w / w)$ within $15 \mathrm{~min}$ of exposure to water [1]. Thus, a fast release of the highly water-soluble peptide desmopressin was therefore expected. Accordingly, approximately $80 \%$ of the encapsulated desmopressin was released fast (within $20 \mathrm{~min}$ ) from the nanofibers, and the complete release of desmopressin, represented by the amount detected in both chambers, was observed after approximately 60 min (Figure $2 b$ ). In the presence of a water-repelling backing film, the unidirectional release of desmopressin from the nanofiber-film hybrid system was achieved, as $<1 \%$ of the encapsulated desmopressin was detected in the left chamber fronting the backing membrane and approximately $80 \%$ of the loaded desmopressin was released within $45 \mathrm{~min}$ to the right receiver chamber fronting the nanofiber layer (Figure $2 \mathrm{~b}$ ). In total, $100 \%$ of desmopressin was released from the fiber-film hybrid system within $1 \mathrm{~h}$ (Figure $2 \mathrm{~b}$ ). This confirms that the nanofiber-film hybrid system indeed ensures the unidirectional and complete release of the encapsulated therapeutic peptide. The average cumulative release of desmopressin exceeded $100 \%$ after $\geq 90 \mathrm{~min}$, but the cumulative release was not statistically significant different from $100 \%$ at any time point. A slight evaporation of the release medium over time at $37^{\circ} \mathrm{C}$ can cause the average cumulative release of desmopressin to exceed $>100 \%$ for some samples. The release of peptide was determined from 2-3 areas of the same electrospun nanofiber mat, and three individual nanofiber mats were evaluated. In general, no significant difference in peptide release was found between the individual areas nor mats, which is indicative of a homogenous distribution of peptide in the electrospun nanofibers.

Fibers

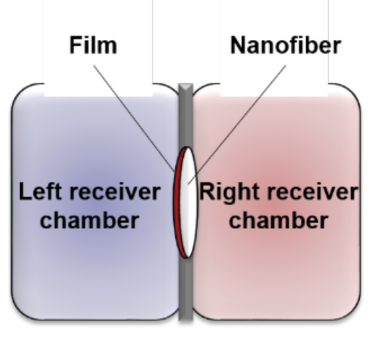

(a)

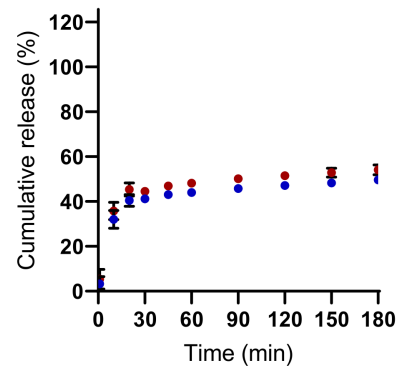

(b)

\section{Fiber-film hybrid system}

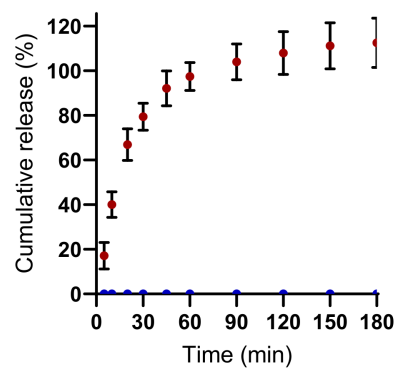

Figure 2. Nanofiber-film hybrid system facilitates unidirectional release of desmopressin. (a) Experimental setup used for studying the release of desmopressin from the nanofibers. The release of desmopressin into both chambers (in the Ussing chamber setup) separated either by the nanofibers or by the nanofiber-film hybrid system was determined. (b) Release of desmopressin from nanofibers or the nanofiber-film hybrid system in the left (blue) and right (red) Ussing chamber, respectively, as depicted in (a). The cumulative release was based on a loading of $8 \%(w / w)$ desmopressin in the chitosan/PEO nanofibers. The data are presented as mean $\pm \mathrm{SD} . N=3, n=2-3$, where $N$ represent the number of individually prepared nanofibers/hybrid systems and $n$ is the number of measurements per system.

\subsection{Nanofiber-Film Hybrid System Protects Peptide from Wash-Out}

Sublingual drug delivery is challenged by a continuous flow of saliva that leads to the wash-out of the drug delivery system and/or drug, which are subsequently swallowed. We have previously demonstrated the good mucoadhesive properties of electrospun chitosan/PEO nanofibers for the sublingual mucosa [8]. We hypothesize that a combination of the mucoadhesive properties of the electrospun chitosan-based nanofibers and the protective function of the backing film will facilitate the close adhesion of the nanofiber-film hybrid system to the sublingual region, and furthermore, protect desmopressin from washout by saliva. The ability of the nanofiber-film hybrid system to prohibit the wash-out of desmopressin was investigated by a flow retention model using ex vivo porcine sublingual 
mucosa (Figure 3a). The flow rate of whole saliva in humans is reported to be around $0.3 \mathrm{~mL} / \mathrm{min}$ without stimulation and up to $7 \mathrm{~mL} / \mathrm{min}$ during stimulation by mastication, etc. [16]. The flow rate used in this experimental setup was $0.5 \mathrm{~mL} / \mathrm{min}$, and thus was within the average salivary flow rate in vivo. Isotonic buffer with $0.05 \%(w / v)$ bovine serum albumin (BSA) with a $\mathrm{pH}$ of 6.8 , which is the average $\mathrm{pH}$ of saliva secreted without stimulation [17], was used as the medium. The mucosa from the ventral side of the porcine tongue was chosen as the surface of adhesion as it, like the human sublingual mucosa, is non-keratinized, it has rete ridges, and its epithelium thickness is similar to that found in man $[18,19]$.

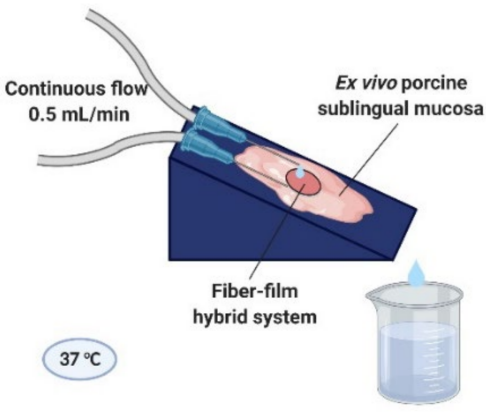

(a)

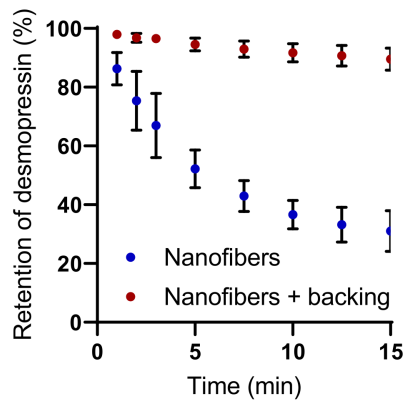

(b)

Figure 3. Nanofiber-film hybrid system protects desmopressin from wash-out by saliva. (a) Ex vivo porcine sublingual mucosa was mounted on a support with an angle of $16^{\circ}$ and the retention of desmopressin on the tissue was evaluated over time. Created by Biorender.com. (b) Retention of desmopressin dosed in nanofibers with and without a saliva-repelling backing film on ex vivo porcine sublingual mucosa. $N=4$, where $\mathrm{N}$ specifies the number of biological replicates.

MiniRin ${ }^{\circledR}$ desmopressin freeze-dried tablets dissolved immediately when exposed to moisture, and thus the tablet disintegrated within seconds and was quickly washed off the ex vivo porcine sublingual mucosa after initiating the flow. The content of desmopressin released from the commercial tablets was not quantified due to the presence of gelatin in the tablets, which interfered with the analysis method. In contrast, the nanofibers, irrespective of the presence of a protective backing film, adhered strongly to the ex vivo porcine sublingual mucosa throughout the duration of the experiment. Desmopressin was quickly released and washed out of the nanofibers without a protective backing-film, al-though it was lost in significantly less time than desmopressin released from MiniRin ${ }^{\circledR}$ freeze-dried tablets. Hence, the mucoadhesive nanofibers alone retained desmopressin significantly longer on the tissue in comparison to the marketed tablet. In accordance with the hypothesis, the nanofiber-film hybrid system further protected desmopressin from wash-out and retained approximately $90 \%$ of the loaded desmopressin on the tissue after 15 min of exposure to flow.

Mucoadhesive drug delivery systems, ensuring unidirectional release, thus not only limit loss of drug by wash-out, but also provide an advantageous increase in the residence time of the drug on the mucosal tissue, the site of absorption. Furthermore, a local environment with a high drug concentration can be achieved between the adhesive drug delivery system and the sublingual mucosa. This increases the concentration gradient of the drug across the mucosal barrier and can lead to improved absorption. Furthermore, the local treatment of, e.g., lesions in the oral mucosa is possible if wash-out is prevented. Site-specific drug delivery can limit exposure to other sites in the oral cavity in general, which is beneficial for drugs with an unpleasant taste and mouthfeel or teeth staining. Some drugs may indeed induce side-effects upon swallowing, which can be avoided if they are efficiently absorbed from the oral mucosa. Thus, electrospinning is an interesting approach to produce solid dosage forms for mucosal application, as this method benefits from short processing times, mild drying conditions and prospects for industrial scalabi-lity, 
continuous manufacturing, as well as reduced cost [20,21]. Additional benefits are that biopharmaceuticals, formulated as solid dosage forms, display improved drug stabi-lity and offer easier handling for patients or healthcare personnel, as well as a reduced cost of transportation [20].

\section{Materials and Methods}

\subsection{Materials}

Chitoceuticals chitosan 95/100 (degree of deacetylation 96\%, Mw 100-250 kDa, chitosan96) was purchased from Heppe Medical Chitosan (Halle, Germany). Polyethylene oxide (Mw 900 kDa, PEO), bovine serum albumin (BSA), acetic acid anhydride, Hank's balanced salt solution (HBSS), Dulbecco's phosphate buffered saline (PBS), glycerol ( $\geq 99 \%)$, tributyl citrate and ethyl cellulose, trifluoroacetic acid (TFA), and acetonitrile were obtained from Sigma Aldrich (St. Louis, MO, USA). N-2-hydroxyethylpiperazine-N'-2-ethanesulfonic acid (HEPES) was obtained from PanReac AppliChem (Darmstadt, Germany). Iron(III) oxide (Secovit ${ }^{\circledR}$ E172) was from BASF (Copenhagen, Denmark). Desmopressin as TFA salt (purity $>98 \%$ ) was obtained from SynPeptide (Shanghai, China). MiniRin ${ }^{\circledR}$ freeze-dryed tablets-60 $\mu \mathrm{g}$ desmopressin (Ferring Pharmaceuticals, Copenhagen, Denmark) were purchased through the Association of Danish Pharmacies. Ultrapure water $(18.2 \mathrm{M} \Omega \times \mathrm{cm})$ purified by a PURELAB flex 4 (ELGA LabWater, High Wycombe, UK) was used.

\subsection{Electrospinning of Chitosan/PEO Nanofibers with Desmopressin}

The chitosan/PEO nanofibers were produced according to Stie et al., 2019 [7] (Figure $4 a)$. Briefly, a $2 \%(w / w)$ clear solution of chitosan in $0.7 \%(w / w)$ acetic acid was prepared in ultrapure water and $4 \%(w / w)$ PEO was dissolved in ultrapure water. Both solutions were stirred for two days at room temperature (RT) to ensure the complete hydration of the polymers. To obtain a $1: 1(w / w)$ ratio of chitosan:PEO and a $8 \%(w / w)$ loading efficiency of the peptide, $1.8 \mathrm{~g}$ of $2 \%(w / w)$ chitosan and $0.9 \mathrm{~g}$ of $4 \%(w / w)$ PEO were mixed and stirred for at least $15 \mathrm{~min}$, and hereafter, mixed with $8 \mathrm{mg}$ desmopressin-TFA (corresponding to $6.6 \mathrm{mg}$ desmopressin). The polymer-peptide solution was stirred for at least $30 \mathrm{~min}$ before electrospinning from a $1 \mathrm{~mL}$ syringe with a $20 \mathrm{G}$ blunt needle (PhotoAdvantage, Ancaster, ON, Canada) by an ES50P-10W high voltage source set to $20 \mathrm{kV}$. The temperature was controlled in the range $23-25^{\circ} \mathrm{C}$ and a low humidity $(<25 \%$ relative humidity) was maintained by a continuous flow of dry air. The nanofibers were collected on aluminum foil on a stainless steel collector plate placed $15 \mathrm{~cm}$ from the tip of the needle. The fibers were stored in a desiccator at $4{ }^{\circ} \mathrm{C}$ to avoid absorption of water and to preserve the stability of desmopressin. Further experiments were conducted within two weeks of the preparation of the electrospun nanofibers.

(a)
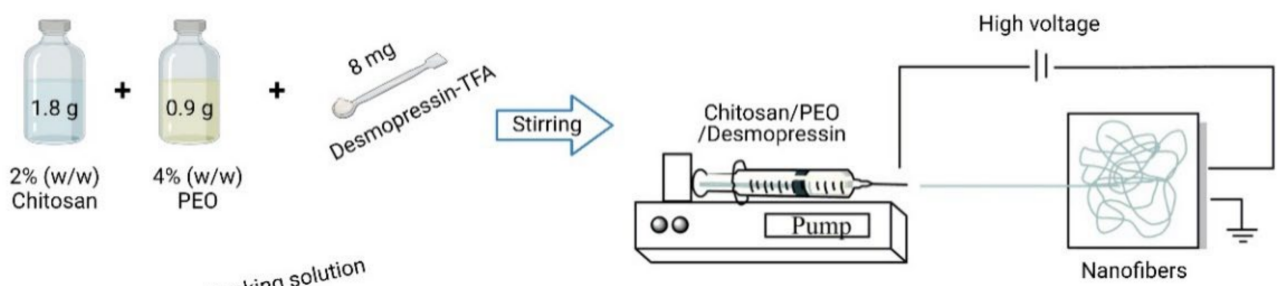

(b)
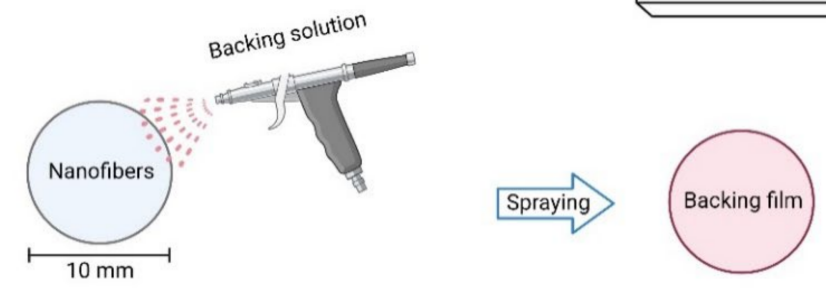

Figure 4. Preparation of the nanofiber-film hybrid system. (a) Electrospinning of chitosan/PEO na-nofibers with desmopressin as described in Section 3.2. (b) Spraying of film on nanofibers as described in Section 3.3. Created by Biorender.com. 


\subsection{Preparation of Nanofiber-Film Hybrid System}

Ten-millimeter discs of peptide-loaded nanofibers were punched out from the main mat and weighed to determine the exact loading of desmopressin per disc. Amounts of $141 \mathrm{mg}$ of acetyl tributyl citrate, $47 \mathrm{mg}$ glycerin, and $750 \mathrm{mg}$ ethyl cellulose were dissolved in $15 \mathrm{~mL}$ ethanol (absolute) and stirred overnight at room temperature. Iron oxide was added and the solution was stirred for at least $30 \mathrm{~min}$. The film was sprayed directly on the $10 \mathrm{~mm}$ discs of nanofibers employing an airbrush (Model BD-134, Custom Colors, Jyderup, Denmark) (Figure $4 \mathrm{~b}$ ). The fibers were fixed on an aluminum plate with holes under vacuum to avoid movement of the fibers as a result of the air flow from the airbrush during the application of the film-forming material by spraying.

\subsection{Visualization of Nanofiber Morphology by Scanning Electron Microscopy}

The morphology of the nanofibers was visualized by scanning electron microscopy (SEM), as previously described by Stie et al., 2019 [7]. The fiber-film hybrid system was cut with a scalpel and mounted vertically on the carbon tape to achieve an image of the cross-section of the fiber-film hybrid system. The average diameter of the nanofibers was determined using the Image J version 1.51j8 software (National Institute of Health, Bethesda, MD, USA) by measuring 25 individual fibers from four areas of interest from a total of three individual batches of electrospun nanofibers loaded with desmopressin.

\subsection{Release of Desmopressin from Fiber-Film Hybrid System in Ussing Chambers}

Discs of nanofibers (diameter $10 \mathrm{~mm}$, weight 2-3 mg) or the nanofiber-film hybrid system were fixed in Ussing sliders with a diffusion area of $0.4 \mathrm{~cm}^{2}$ and placed in a side-byside diffusion cells apparatus EM-CSY-8 Ussing chambers (Physiologic Instruments, San Diego, CA, USA), with $2 \mathrm{~mL}$ of $10 \mathrm{mM}$ HEPES in HBSS pH 6.8 with $0.05 \%(w / v)$ BSA in each chamber, and incubated at $37^{\circ} \mathrm{C}$ for $3 \mathrm{~h}$. Samples of $100 \mu \mathrm{L}$ were collected from each chamber at various time intervals and replenished with $100 \mu \mathrm{L}$ warm $10 \mathrm{mM}$ HEPES in HBSS pH 6.8 with $0.05 \%(w / v)$ BSA. The samples were centrifuged $(10,000 \mathrm{rpm} / 9279 \times g$, $10 \mathrm{~min}, 5^{\circ} \mathrm{C}$ ) and the concentration of desmopressin was determined by high-performance liquid chromatography with UV detection (HPLC-UV, Experimental Section 3.7). The cumulative release of desmopressin (M) was determined according to Equation (1).

$$
M=V_{S} \cdot\left(\sum_{n=1}^{n} C_{n-1}\right)+C_{n} \cdot V_{T}
$$

where $V_{S}$ is the sample volume $(100 \mu \mathrm{L}), C_{n}$ is the concentration of desmopressin at time point $n$, and $\mathrm{V}_{\mathrm{T}}$ is the total volume of the receiver Ussing chambers $(2 \mathrm{~mL})$. The cumulative peptide release in percent $(\%)$ was calculated based on the theoretical loading of desmopressin of $8 \%(w / w)$ and a diffusion area of $0.4 \mathrm{~cm}^{2}$.

\subsection{Ex Vivo Flow Retention Model}

The ex vivo flow retention setup was inspired by Madsen et al. [17]. Sublingual porcine tissue from healthy pigs (approximately 30-60 kg, Danish Landrace/Yorkshire/Duroc) was collected immediately after euthanization and kept in PBS on ice until use on the same day as harvesting of the tissue. Thin sections $(0.5-0.7 \mathrm{~mm})$ of the ventral side of the tongue were obtained by means of an electric dermatome (Zimmer Biomet, Alberts-lund, Denmark). The sublingual mucosa was mounted on a rubber pad with pins at an angle of $16^{\circ}$ and placed on a heating plate to achieve a temperature of approximately $37^{\circ} \mathrm{C}$ of the tissue. The ex vivo sublingual mucosa was equilibrated for $10-15 \mathrm{~min}$ in warm $\left(37^{\circ} \mathrm{C}\right) 10 \mathrm{mM}$ HEPES in HBSS pH 6.8 with $0.05 \%(w / v)$ BSA with a flow of $0.5 \mathrm{~mL} / \mathrm{min}$ from two $13 \mathrm{G}$ needles placed above the tissue and the flow was controlled by a syringe pump (11 Elite, Harvard Apparatus, Holliston, MA, USA). $10 \mathrm{~mm}$ discs of electrospun nanofibers, with or without backing, or a MiniRin ${ }^{\circledR}$ freeze-dried tablet ( $60 \mu \mathrm{g}$ desmopressin) were placed on the mucosal tissue and flushed with warm $\left(37^{\circ} \mathrm{C}\right) 10 \mathrm{mM}$ HEPES in HBSS with $0.05 \%(w / v)$ 
BSA, pH 6.8 with a flow of $0.5 \mathrm{~mL} / \mathrm{min}$ for $15 \mathrm{~min}$ controlled by a syringe pump (11 Elite, Harvard Apparatus, Holliston, MA, USA). Samples of $100 \mu \mathrm{L}$ of the eluate were collected after $1,2,3,5,7.5,10,12.5$ and $15 \mathrm{~min}$ and centrifuged $(10,000 \mathrm{rpm} / 9279 \times \mathrm{g}, 10 \mathrm{~min}$, $5{ }^{\circ} \mathrm{C}$ ). The concentration of desmopressin was determined by HPLC-UV (Experimental Section 3.7). As gelatin from the MiniRin ${ }^{\circledR}$ tablets interfered with the HPLC-UV method, the concentration of desmopressin released from the freeze-dried tablets was not determined. The retention of desmopressin was determined according to Equation (2).

$$
\text { Peptide wash out }(m g)=V_{S} \cdot \sum_{n=1}^{n} C_{n-1}+C_{n} \cdot\left(V_{T}-\sum_{n=1}^{n} V_{n-1}\right)
$$

where $V_{S}$ is the eluate sample volume $(100 \mu \mathrm{L}), C_{n}$ is the concentration of desmopressin at time point $n, \mathrm{~V}_{\mathrm{T}}$ is the total volume of eluate at time point $n$ based on a flow of $0.5 \mathrm{~mL} / \mathrm{min}$, and $\sum_{n=1}^{\mathrm{n}} \mathrm{V}_{\mathrm{n}-1}$ is the sum of the eluate volumes sampled at time point $n-1$. The retention of desmopressin in percent (\%) was determined based on a theoretical loading of $8 \%(100 \%$ peptide retention) $(w / w)$.

\subsection{Quantification of Desmopressin by HPLC-UV}

Desmopressin was quantified by HPLC-UV $(\lambda 218 \mathrm{~nm})$ on a Shimadzu Prominence system (Kyoto, Japan) with an Aeris peptide XB-C18 column $(100 \times 2.1 \mathrm{~mm}, 3.6 \mu \mathrm{m}$, Phenomenex, Torrance, CA, USA). Desmopressin was eluted from $10 \mu \mathrm{L}$ samples with a gradient $0 \rightarrow 40 \%$ eluent $B$ in eluent $A$ over $8 \mathrm{~min}$ at $0.8 \mathrm{~mL} / \mathrm{min}$ at $40{ }^{\circ} \mathrm{C}$, where eluent $\mathrm{A}$ consisted of 95:5:0.1\% $(v / v)$ acetonitrile:water:TFA and eluent B of 5:95:0.1\% $(v / v)$ acetonitrile:water:TFA. The samples were stored at $4{ }^{\circ} \mathrm{C}$ during analysis. The limit of detection and limit of quantification were $0.09 \mu \mathrm{g} / \mathrm{mL}$ and $0.26 \mu \mathrm{g} / \mathrm{mL}$, respectively.

\section{Conclusions}

The therapeutic peptide desmopressin was encapsulated within mucoadhesive electrospun chitosan/PEO nanofibers intended for sublingual delivery. A two-layered hybrid drug delivery system was developed by combining a saliva-repelling backing film with the nanofibers to ensure unidirectional drug release. The nanofibers displayed a unidirectional, controlled, and fast release of desmopressin with an approximately $80 \%$ release of the loaded peptide from the nanofiber-based hybrid system within $45 \mathrm{~min}$. Importantly, the nanofiber-film hybrid system showed resilience to saliva flow and retained approximately $90 \%$ of the desmopressin loaded on the tissue after $15 \mathrm{~min}$ of exposure to flow. This can potentially improve the absorption of peptide, but also potentially improve the absorption of small molecular drugs across the mucosae. Mucoadhesive electrospun na-nofibers are considered promising carriers for peptide delivery via mucosal routes upon, e.g., sublingual administration.

Author Contributions: Conceptualization, M.B.S., I.S.C., J.J. and H.M.N.; methodology, M.B.S., J.R.G., I.S.C., J.J. and H.M.N.; investigation, M.B.S. and J.R.G.; resources, I.S.C., J.J. and H.M.N.; writingoriginal draft preparation, M.B.S.; writing-review and editing, J.R.G., I.S.C., J.J. and H.M.N.; funding acquisition, I.S.C., J.J. and H.M.N. All authors have read and agreed to the published version of the manuscript.

Funding: This research was funded by The Danish Council for Independent Research, Technology and Production, grant number DFF-6111-00333; the Novo Nordisk Foundation Grand Challenge Program, NNF16OC0021948; and Innovation Fund Denmark PROBIO, project-7076-00053B.

Institutional Review Board Statement: Not applicable.

Informed Consent Statement: Not applicable.

Data Availability Statement: The data presented in this study are available on request from the corresponding author. 


\begin{abstract}
Acknowledgments: Department of Experimental Medicine and the Large Animal Teaching Hospital at University of Copenhagen are acknowledged for assisting with the porcine tissue used for the ex vivo studies. The authors acknowledge LEO Pharma (Ballerup, Denmark) for granting the use of the dermatome equipment.
\end{abstract}

Conflicts of Interest: The authors declare no conflict of interest.

\title{
References
}

1. Walsh, G. Biopharmaceutical benchmarks 2018. Nat. Biotechnol. 2018, 36, 1136-1145. [CrossRef] [PubMed]

2. Mitragotri, S.; Burke, P.A.; Langer, R. Overcoming the challenges in administering biopharmaceuticals: Formulation and delivery strategies. Nat. Rev. Drug Discov. 2014, 13, 655-672. [CrossRef] [PubMed]

3. Kraan, H.; Vrieling, H.; Czerkinsky, C.; Jiskoot, W.; Kersten, G.; Amorij, J.-P. Buccal and sublingual vaccine delivery. J. Control. Release 2014, 190, 580-592. [CrossRef] [PubMed]

4. Rathbone, M.J.; Drummond, B.K.; Tucker, I.G. The oral cavity as a site for systemic drug delivery. Adv. Drug Deliv. Rev. 1994, 13, 1-22. [CrossRef]

5. Schiele, J.T.; Quinzler, R.; Klimm, H.-D.; Pruszydlo, M.G.; Haefeli, W.E. Difficulties swallowing solid oral dosage forms in a general practice population: Prevalence, causes, and relationship to dosage forms. Eur. J. Clin. Pharmacol. 2013, 69, 937-948. [CrossRef]

6. Gandhi, R.B.; Robinson, J.R. Oral cavity as a site for bioadhesive drug delivery. Adv. Drug Deliv. Rev. 1994, 13, 43-74. [CrossRef]

7. Stie, M.B.; Jones, M.; Sørensen, H.O.; Jacobsen, J.; Chronakis, I.S.; Nielsen, H.M. Acids 'generally recognized as safe' affect morphology and biocompatibility of electrospun chitosan/polyethylene oxide nanofibers. Carbohydr. Polym. 2019, 215, 253-262. [CrossRef]

8. Stie, M.B.; Gätke, J.R.; Wan, F.; Chronakis, I.S.; Jacobsen, J.; Nielsen, H.M. Swelling of mucoadhesive electrospun chitosan/polyethylene oxide nanofibers facilitates adhesion to the sublingual mucosa. Carbohydr. Polym. 2020, 242, 116428. [CrossRef]

9. Sharma, A.; Gupta, A.; Rath, G.; Goyal, A.; Mathur, R.B.; Dhakate, S.R. Electrospun composite nanofiber-based transmucosal patch for anti-diabetic drug delivery. J. Mater. Chem. B 2013, 1, 3410-3418. [CrossRef]

10. Xu, L.; Sheybani, N.; Ren, S.; Bowlin, G.L.; Yeudall, W.A.; Yang, H. Semi-Interpenetrating Network (sIPN) Co-Electrospun Gelatin/Insulin Fiber Formulation for Transbuccal Insulin Delivery. Pharm. Res. 2014, 32, 275-285. [CrossRef]

11. Lancina, M.G.; Shankar, R.K.; Yang, H. Chitosan nanofibers for transbuccal insulin delivery. J. Biomed. Mater. Res. Part A 2017, 105, 1252-1259. [CrossRef] [PubMed]

12. Berka, P.; Stránská, D.; Semecký, V.; Berka, K.; Doležal, P. In vitro testing of flash-frozen sublingual membranes for storage and reproducible permeability studies of macromolecular drugs from solution or nanofiber mats. Int. J. Pharm. 2019, 572, 118711. [CrossRef] [PubMed]

13. Edmans, J.G.; Murdoch, C.; Santocildes-Romero, M.E.; Hatton, P.V.; Colley, H.E.; Spain, S.G. Incorporation of lysozyme into a mucoadhesive electrospun patch for rapid protein delivery to the oral mucosa. Mater. Sci. Eng. C 2020, 112, 110917. [CrossRef] [PubMed]

14. Van Kerrebroeck, P.; Nørgaard, J.P. Desmopressin for the treatment of primary nocturnal enuresis. Ped. Health 2009, 3, 311-327. [CrossRef]

15. Sharman, A.; Low, J. Vasopressin and its role in critical care. Contin. Educ. Anaesth. Crit. Care Pain 2008, 8, 134-137. [CrossRef]

16. Iorgulescu, G. Saliva between normal and pathological. Important factors in determining systemic and oral health. J. Med. Life 2009, 2, 303-3007.

17. Madsen, K.D.; Sander, C.; Baldursdottir, S.; Pedersen, A.M.L.; Jacobsen, J. Development of an ex vivo retention model simulating bioadhesion in the oral cavity using human saliva and physiologically relevant irrigation media. Int. J. Pharm. 2013, 448, 373-381. [CrossRef]

18. Thirion-Delalande, C.; Gervais, F.; Fisch, C.; Cuiné, J.; Baron-Bodo, V.; Moingeon, P.; Mascarell, L. Comparative analysis of the oral mucosae from rodents and non-rodents: Application to the nonclinical evaluation of sublingual immunotherapy products. PLoS ONE 2017, 12, e0183398. [CrossRef]

19. Kondo, M.; Yamato, M.; Takagi, R.; Murakami, D.; Namiki, H.; Okano, T. Significantly different proliferative potential of oral mucosal epithelial cells between six animal species. J. Biomed. Mater. Res. Part A 2014, 102, 1829-1837. [CrossRef]

20. Vass, P.; Démuth, B.; Hirsch, E.; Nagy, B.; Andersen, S.K.; Vigh, T.; Verreck, G.; Csontos, I.; Nagy, Z.K.; Marosi, G. Drying technology strategies for colon-targeted oral delivery of biopharmaceuticals. J. Control. Release 2019, 296, 162-178. [CrossRef]

21. Vass, P.; Démuth, B.; Farkas, A.; Hirsch, E.; Szabó, E.; Nagy, B.; Andersen, S.K.; Vigh, T.; Verreck, G.; Csontos, I.; et al. Continuous alternative to freeze drying: Manufacturing of cyclodextrin-based reconstitution powder from aqueous solution using scaled-up electrospinning. J. Control. Release 2019, 298, 120-127. [CrossRef] [PubMed] 